\title{
Research and Development Work on Lithium-ion Batteries for Environmental Vehicles
}

\author{
Osamu Shimamura*, Takaaki Abe*, \\ Kyoichi Watanabe*, Yasuhiko Ohsawa*, Hideaki Horie*
}

\begin{abstract}
Interest in electric vehicles (EVs) and hybrid electric vehicles (HEVs) has risen dramatically on account of environmental and energy concerns. The biggest issues that must be addressed in order to popularize these advanced vehicles are related to the battery.

We have been promoting vigorous $\mathrm{R} \& \mathrm{D}$ work on batteries for application to environmental vehicles since the beginning of the 1990s. Attention was focused on lithium-ion batteries early on as a fundamental solution to the critical issue mentioned above. The conclusion was reached that the development of those potentialities to their fullest extent would create completely new forms of value unobtainable with conventional batteries. As a result of extensive theoretical studies and many experimental demonstrations, we successfully showed ahead of others that those potentialities did in fact exist and could be achieved.

This paper makes clear the various performance requirements of advanced batteries for EV or HEV application, focusing in particular on the critical aspects of the battery thermal design and construction for system stability. It also explains how the power output of the lithium-ion battery has been substantially improved for application to HEVs.
\end{abstract}

Keywords: Hybrid Electric Vehicles, Electric Vehicles, Li-ion Battery

\section{INTRODUCTION}

Research and development efforts are under way to put environmental vehicles on the market. Among the powertrain issues that must be addressed in connection with these advanced vehicles are to secure sufficient power output to satisfy the power performance required by the vehicle and to raise energy efficiency to the highest level possible. Toward that end, the performance of the powertrain components must be enhanced and resistance losses must be thoroughly reduced.

We have been focusing on lithium-ion battery systems because of their inherent potential for building future power sources for environmental vehicles. Since 1992, a full-scale program has been under way to research and develop lithium-ion batteries suitable to each type of environmental vehicle. ${ }^{1-15}$ In that process, we demonstrated for the first time anywhere that lithium-ion batteries are outstanding battery systems not only in terms of capacity, but also with respect to their high power characteristics. ${ }^{16-21}$

In conducting concrete studies concerning the applicability of lithium-ion batteries to different vehicle

* NISSAN MOTOR CO., LTD. Nissan research center, Electric Propulsion Laboratory, 1 Natsushima, Yokosuka, Kanagawa 237-8523, Japan types, optimization of battery capacity and output was regarded as being the first step. A key factor in studying power output is to analyze internal battery resistance, especially from the perspectives of the reactions that take place at the electrodes and electron and ion transport. Reducing internal resistance improves battery output, enhances energy efficiency and suppresses heat generation inside the battery, which, in turn, leads to higher reliability.

One prerequisite for vehicle application is a suitable thermal design that takes into account the conditions of the usage environment, because it is presumed that the battery will undergo continuous large-current charging/discharging over a long period of time. Our research has also shown that lithium-ion batteries allow an excellent thermal design to meet this requirement. As a result, a compact power source system can be constructed that can be expected to improve vehicle mountability.

This paper discusses lithium-ion batteries from the dual perspectives of battery performance optimization and system feasibility, including the thermal design, with regard to their use on different types of environmental vehicles. 


\section{OUTPUT CHARACTARISTICS OF LITHIUM-ION BATTERYS}

\subsection{History of Improvement in Secondary Battery Performance}

There are two fundamental indices of battery performance: (1) energy density that indicates how much energy a battery can store and (2) power density that indicates its maximum output. For example, in the case of electric vehicles (EVs), the driving range is dependent on the battery's energy storage capacity, which calls into question the magnitude of the battery's energy density. For hybrid electric vehicles (HEVs), on the other hand, which are fitted with a generator, the ability to supply power for acceleration and to accept regenerated power during deceleration is more important than the quantity of energy the battery can store. Figure 1 shows a historical outline of the improvement achieved in the energy density of typical secondary batteries for use in electric appliances.

Prior to the 1990s, lead-acid batteries and nickel-cadmium batteries were representative examples of secondary batteries. The energy density of nickel-cadmium batteries did not exceed approximately $50 \mathrm{Wh} / \mathrm{kg}$ at the most. In the early $1990 \mathrm{~s}$, nickel-metal hydride batteries and lithium-ion batteries appeared in succession, and the energy density of small batteries for consumer electronics products has been improved by nearly fourfold in roughly the last 15 years.

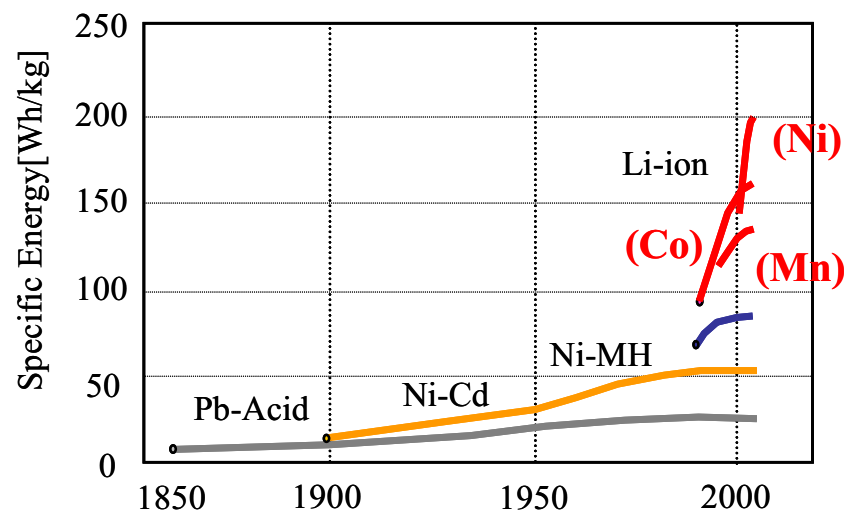

Fig. 1 Specific energy of secondary batteries for electric appliances

\subsection{Energy Consumption Inside Batteries}

The consumption of energy inside a battery consists of the portion attributable to battery reactions and energy losses accompanying charge transport, i.e., either electron conduction or ion conduction. The smaller these values are, the less energy that is consumed inside the battery, which reduces internal resistance and increases the battery's intrinsic output voltage. With regard to the electrode structure, reducing the thickness of the active material layers lowers resistance to the passage of lithium ions and electrons through the layers. However, that would gradually increase the relative share of energy consumption accounted for by components not directly involved in energy storage, such as the separators or current collectors, which would cause the battery's energy density to decline.

\subsection{Battery Output Simulation}

More accurate predictions of internal resistance values in a battery are needed in order to ascertain internal energy consumption more precisely. Resistance values are mutually dependent on such variables as the ion concentration and current value. Accordingly, they can only be obtained by solving equations consisting of many related physical quantities. Based on this way of thinking, we constructed a battery output simulation model that combines battery reactions and charge transport, as shown in Fig. 2. ${ }^{22-23}$ This model consists of two fundamental schemes for calculating charge transport phenomena: (1) lithium-ion diffusion equations for calculating the parameters mutually dependent on the active materials and electrolyte and

(2) electron conduction equations.
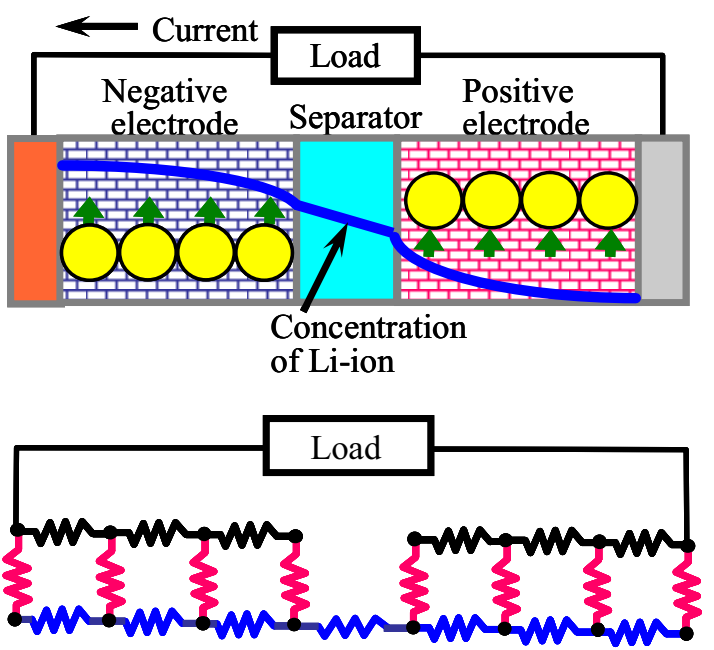

Fig. 2 Battery simulation combined with lithium diffusion and electron conductivity equations

(1) Lithium-ion diffusion equations

Equation for lithium-ion transport in active materials $\frac{\partial C_{s}}{\partial t}=D_{s} \nabla_{r}^{2} \cdot C_{s}$

Equation for lithium-ion transport in electrolyte

$$
\varepsilon \frac{\partial C_{e}}{\partial t}=\varepsilon D_{e} \nabla_{X}^{2} \cdot C_{e}+\frac{\left(1-t_{+}\right)}{F} a i_{n}
$$

Equation for current transfer in active materials

$$
\sigma_{s} \nabla_{X}^{2} \cdot \phi_{s}-a i_{n}=0
$$

(2) Electron conduction equations

Equation for current transfer in electrolyte

$$
\begin{aligned}
& \sigma_{e} \nabla_{X}{ }^{2} \cdot \phi_{e}+a i_{n}-\sigma_{e} \nabla_{X}{ }^{2} \cdot\left(\ln C_{e}\right) \cdot 2 R T\left(1-t_{+}\right)=0 \\
& i_{n}=k C_{e}^{\alpha a}\left(C_{t}-C_{s}\right)^{\alpha a} C_{s}^{\alpha c}\left\{\exp \left(\frac{\alpha F}{R T} \times \eta\right)-\exp \left(-\frac{\alpha F}{R T} \times \eta\right)\right\} \\
& \eta=\phi_{S}-\phi_{e}-U\left(C_{S}\right)
\end{aligned}
$$




\section{List of symbols}

C : concentration of Lithium ion

$\mathrm{D}$ : diffusion coefficient of Lithium ion

$\mathrm{r}$ : radial distance in a particle of active material

$\mathrm{X}$ : distance from the negative electrode current collector

$\varepsilon$ : prosity

$\mathrm{t}_{+}:$trancsference number of cation

F : Faraday's constant

a : surface rate of active material

$i_{n}$ : current density

$\sigma:$ conductivity

$\phi$ : potential

$\mathrm{R}$ : universal gas constant

$\mathrm{T}$ : temperature

$\mathrm{k}$ : reaction rate constant

$\eta$ : overpotential

$\alpha \mathrm{a}, \alpha \mathrm{c}$ : transfer coefficients

$\mathrm{U}$ : open-circuit potential

\section{Subscripts \\ $\mathrm{s}:$ active material \\ e : electrolyte}

In order to validate this simulation model, prototype electrodes were fabricated and evaluated in tests. A comparison was then made of the experimental and calculated results. As an example, Figure 3 compares the calculated and experimental discharged capacity for two different discharge current levels. Good agreement is seen between the experimental and calculated values.

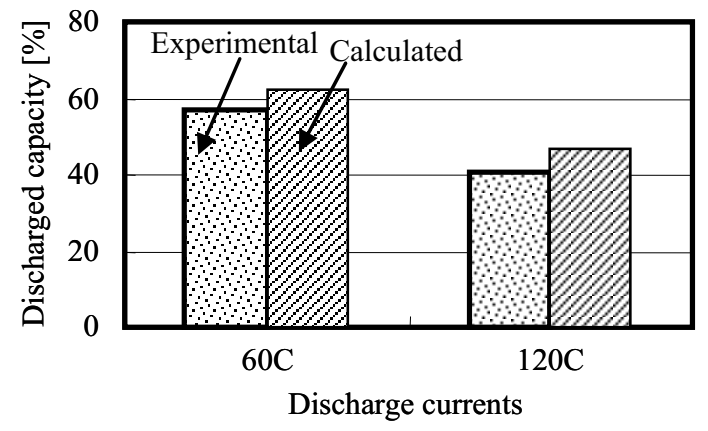

Fig. 3 Comparison of experimental and calculated discharge capacity

\subsection{Optimization of Battery Output and Capacity}

Based on the foregoing insights, the relationship between battery output and capacity was examined. Figure 4 shows the discharged capacity as a function of the electrode thickness. The results indicate that the discharged capacity at the same discharge current decreases with increasing electrode thickness.

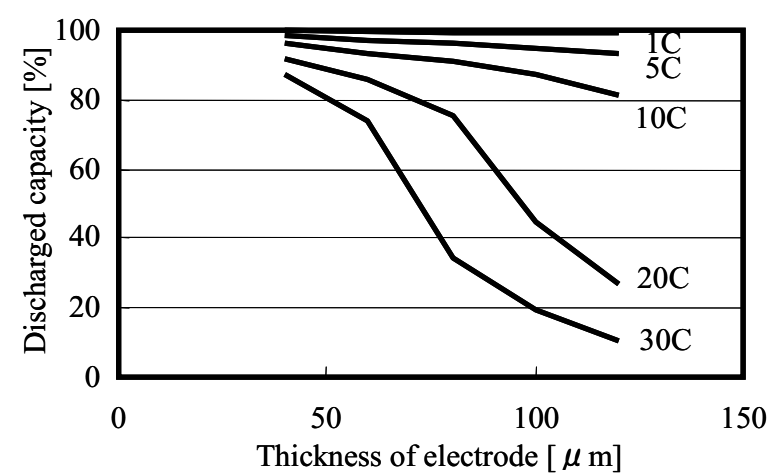

Fig. 4 Discharged capacity with various electrode thicknesses

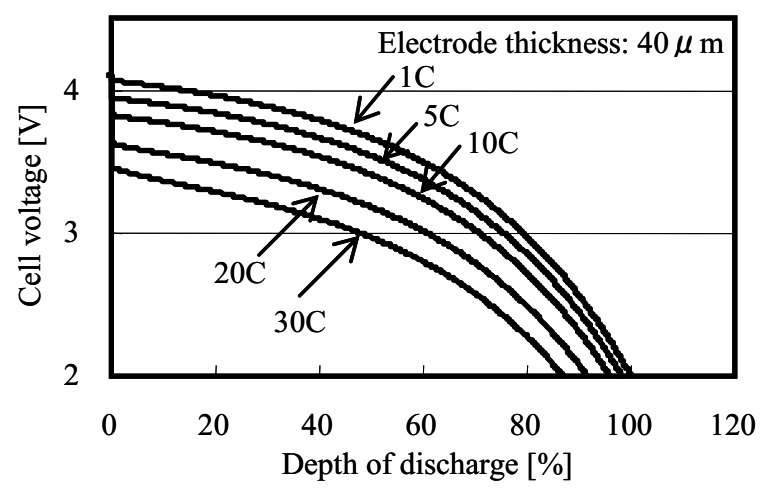

Fig. 5 Voltage drop with various discharge currents

In order to investigate the reason for that decline, the change in the battery voltage during discharge was calculated under the same current conditions as in Fig. 5. The results are shown in Figs. 5-7 for different electrode thicknesses. The results indicate that following the decrease in voltage right after the start of discharge, the terminal voltage of the battery gradually declines and that the difference in this voltage drop increases with a larger discharge current. The main reason for the decline in the maximum output obtainable from the battery is attributed to this terminal voltage drop. It is presumed that in the region where a relatively large current value is demanded, lithium-ion diffusion in the electrodes cannot follow the required discharge current, giving rise to an ion concentration gradient that results in a voltage drop. 


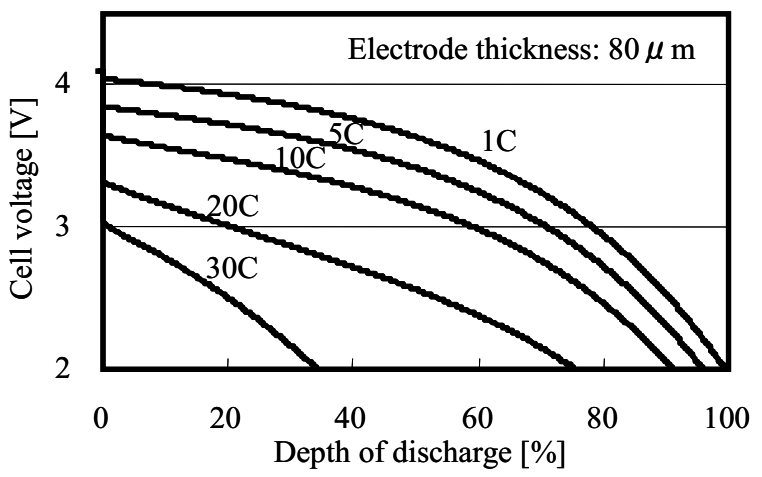

Fig. 6 Voltage drop with various discharge currents

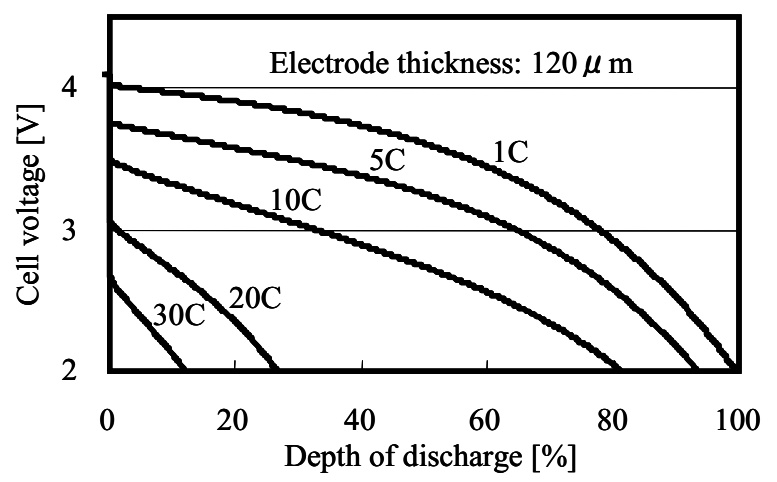

Fig.7 Voltage drop with various discharge currents

Based on the foregoing investigation, the relationship between the battery's maximum output and energy density was simulated, and the results are given in Fig. 8 . This relationship was calculated for different types of vehicles using the same fixed material property parameters. For EVs or different types of HEV systems, the relationship between the battery's maximum output and capacity differs depending on the vehicle's driving range and its power source. As the results in the figure indicate, lithium-ion batteries achieve a good balance between power output and capacity over a wide usage range as a result of optimizing the electrode structure.

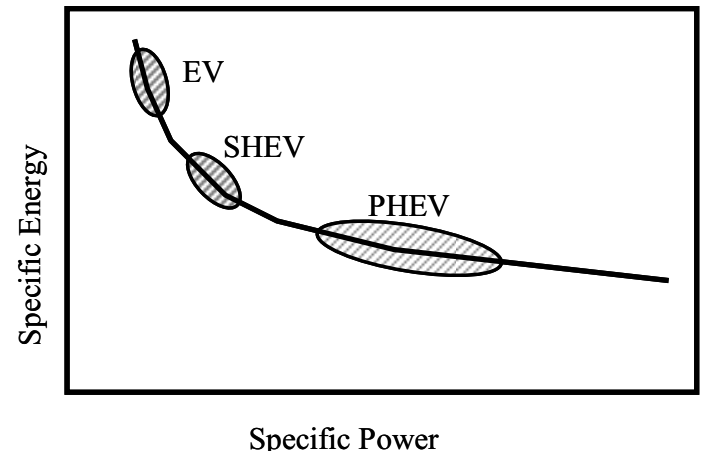

Fig. 8 Relationship between specific energy and specific power (calculated)

\section{THERMAL DESIGN OF LITHIUM-ION BATTERY SYSTEMS}

\subsection{Thermal Behavior of Battery Systems}

Not only does the level of the required discharge current differ among various types environmental vehicles, the charge/discharge frequency and continued duration also vary considerably. The amount of heat generated by the battery increases as the output power demanded by the powertrain increases. Consequently, besides securing the necessary power output and capacity, the battery system design must also ensure that the system is thermally feasible.

Heat generation inside a battery can be attributed to Joule heat originating from internal resistance accompanying charging/discharging processes and the heat of reaction produced by charging/discharging reactions. Such heat generation raises the battery temperature and continued exposure to high temperatures might advance battery degradation. To avoid that possibility, the rise in the battery temperature must be suppressed by applying a suitable cooling system.

\subsection{EV and HEV Driving Modes}

The operating conditions of a battery system differ substantially depending on the type of environmental vehicle involved. In an $\mathrm{EV}$, the battery discharges continuously, but the required specific power relative to the battery capacity (i.e., power-to-energy $(\mathrm{P} / \mathrm{E})$ ratio) is small. In a series hybrid (SHEV), assuming that the battery provides the power to propel the vehicle, the influence of heat generation increases because a smaller battery pack than that of an EV discharges current to drive the traction motor. Additionally, the battery must be able to accept large charge current during a short period of time, which is another aspect that increases the importance of the thermal design. In a parallel hybrid (PHEV), the battery must be able to discharge and accept large currents instantaneously to support vehicle acceleration/deceleration. However, the battery operating time is shorter and the absolute energy quantities are smaller, thus the $\mathrm{P} / \mathrm{E}$ ratio is larger. Large heat generation occurs during charging/discharging because of the large currents involved, but there are long periods of time when the battery is not charging or discharging and can be cooled during such intervals.

Figure 9 shows an example of battery charging/discharging operations during EV, SHEV and PHEV driving modes. Many different scenarios can be considered for the SHEV driving mode. The pattern shown in the figure is for a pure EV mode initially, followed by operation of the generator once the battery's state of charge falls to a certain level. 


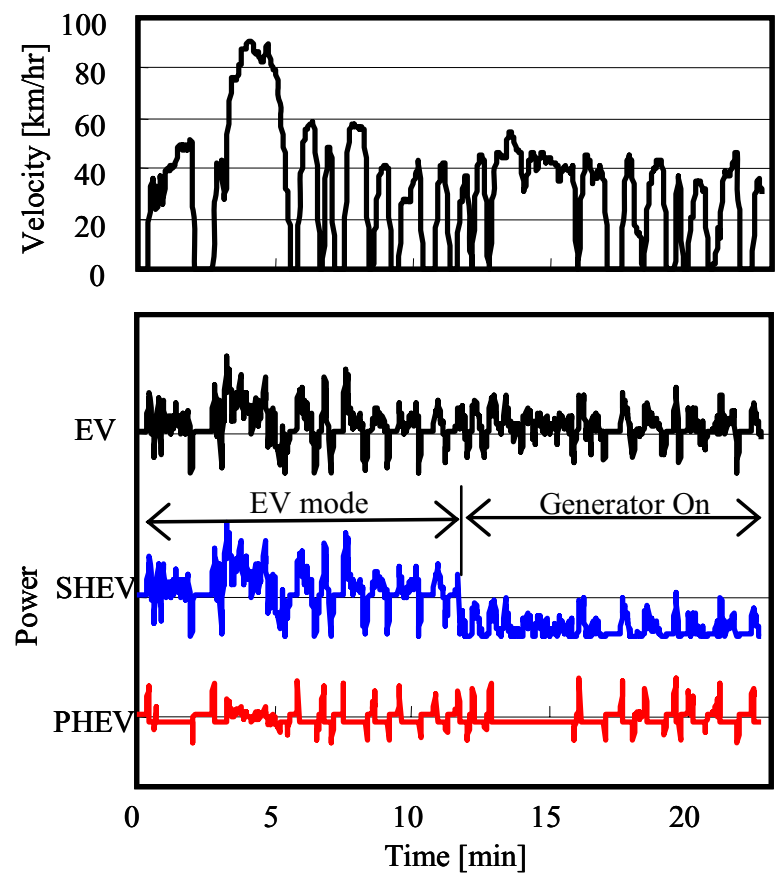

Fig. 9 Driving modes

\subsection{Thermal Characteristics of Batteries}

A study was made of the thermal characteristics of lithium-ion batteries in relation to the various usage conditions of the above-mentioned environmental vehicles. The results are shown in Fig. 10. First, we considered a driving mode in which the battery continues to discharge and accept relatively large currents. The calculated rise in the battery temperature under that condition is shown by the left-side bar graphs in the figure. Each graph shows the equilibrium temperature rise found by dividing the battery heat generation under the operating conditions of each vehicle by the heat radiation coefficient and heat radiation area. It is seen that the PHEV battery, which must discharge a large current in this driving mode, shows the highest temperature rise, indicating a severe thermal condition. However, in order to estimate the actual battery temperature rise, it is necessary to consider the charging time, discharging time and rest time required of each system under this driving mode. The hatched bar graphs on the right side in the figure show the temperature rise that was calculated when these factors were taken into consideration. The graphs show the largest battery temperature rise that occurs in this driving mode. As the results indicate, although the PHEV battery discharges large currents, it has long periods when charging/discharging operations are suspended, thereby increasing the cooling effect. The SHEV battery has long periods of large-current charging/discharging during this driving mode, which create a harsh thermal condition. However, the results of this calculation indicate that this battery design is also thermally feasible.

The change in the battery temperature rise in each vehicle during this driving mode is shown in Fig. 11.
The peak temperature rise in each case represents the right-hand bar graphs in Fig. 10.

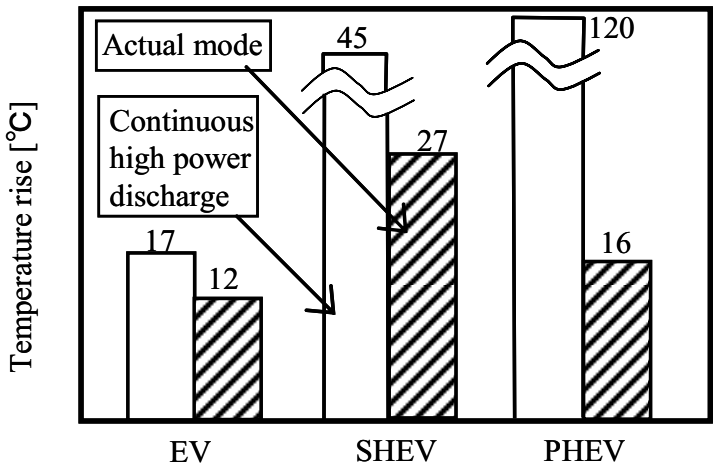

Fig. 10 Battery temperature rise with various vehicles

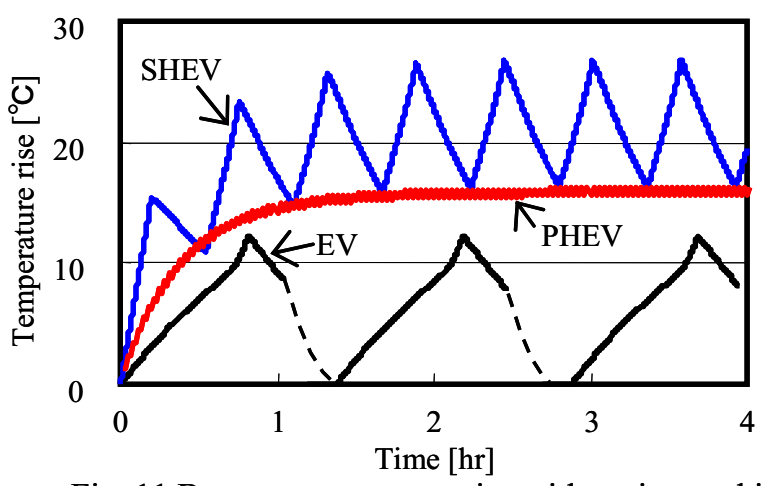

Fig. 11 Battery temperature rise with various vehicles

\subsection{Thermal Characteristics of Aqueous Electrolyte Batteries}

The foregoing sections have presented the results for lithium-ion batteries that use an organic solution as the electrolyte. Heat generation in lithium-ion batteries during charging/discharging can be divided between the heat of reaction accompanying electrochemical reactions and Joule heat induced by internal resistance. However, for batteries that use an aqueous solution as the electrolyte, it is also necessary to consider the electrolysis (and recombination) of water that serves as the solvent. Near the end of charging or when the battery temperature rises, among other situations, the electrolysis reaction may be promoted, causing the battery temperature to increase further.

This concept is outlined in Fig. 12, where the battery temperature is indicated along the horizontal axis. The straight line in the graph expresses heat radiation from the battery surface, and the points where it intersects the battery's heat generation curve indicate the equilibrium temperature. There are three such points of intersection in this figure. The point of intersection in the center of the graph is an unstable solution, whereas the points on the low temperature side (left) and the high temperature side (right) are stable solutions. If the battery temperature is lower than the second solution in the center, it converges to the stable solution on the low 
temperature side; if it is higher, it converges to the solution on the high temperature side. It will be noted that there is zero heat radiation at the point of intersection of the straight line and the $\mathrm{x}$-axis. In other words, that point indicates the ambient temperature, which means that moving the straight line to the left or right changes the ambient temperature. As a result, depending on the operating conditions, there is a possibility that the equilibrium temperature might jump all at once from the low to the high temperature side.

Figure 13 shows an example of the battery temperature calculated for an aqueous solvent electrolyte in an SHEV application. While this is strictly one calculation example, it suggests that the battery temperature might rise sharply when the heat of the water electrolysis reaction is added in. Because of their very nature, lithium-ion batteries do not experience such temperature instability.

The foregoing results have shown that varying the characteristics of lithium-ion batteries makes them suitable for use on all types of environmental vehicles, from EVs to PHEVs, with respect to both power output and thermal feasibility.

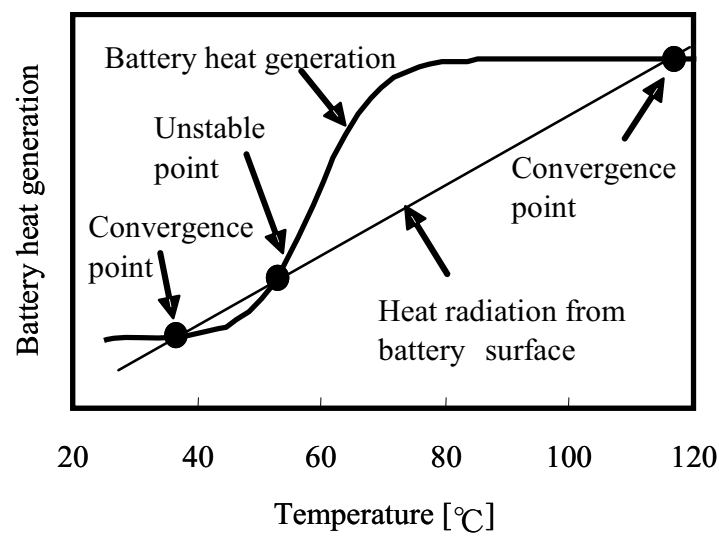

Fig. 12 Analysis of battery equilibrium temperature for medium-size to large batteries

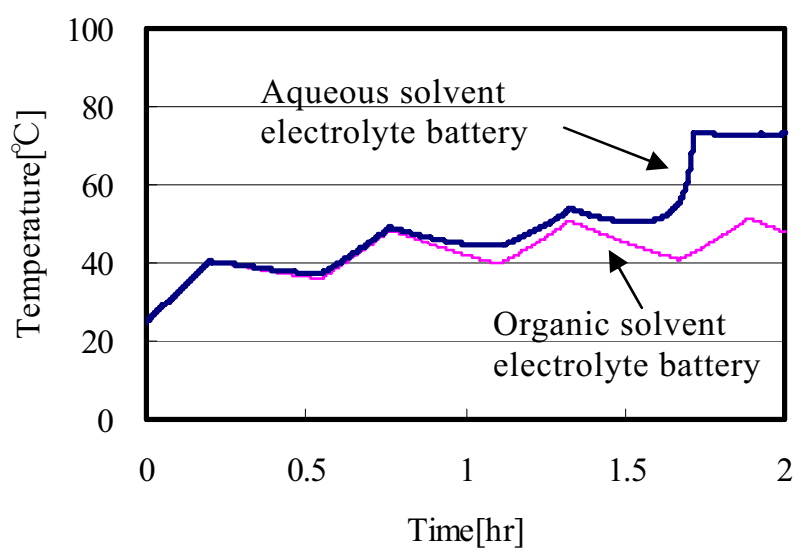

Fig. 13 Comparison of battery temperatures in SHEV application

\section{CONCLUSION}

A simulation model combining electrode reactions and charge transport was constructed for the purpose of predicting battery performance and was used to examine power output and capacity in particular. It was shown that changing the electrode parameters of lithium-ion batteries makes it possible to create power source systems that are suitable for application to a wide range of envisioned environmental vehicles. Furthermore, when used in combination with thermal simulations, it is possible to examine battery systems from the perspective of their thermal design as well. The results of the present study have shown that the examined battery systems are sufficiently feasible for practical use. Accordingly, it is concluded that lithium-ion battery systems are the power sources that are best suited for use on a wide variety of environmental vehicles.

\section{REFERENCES}

[1] H. Horie, et al., Development of a Lithium-ion Battery System for EV Application, Preprints of the Spring Scientific Lecture Series of JSAE, 961 (1996) (in Japanese)

[2] T. Miyamoto, et al., Advanced Battery System for Electric Vehicle (FEV-II), EVS13 (1996).

[3] H. Horie, et al., Development of a High-power Lithium-ion Battery System for HEV Application, Preprints of the Spring Scientific Lecture Series of JSAE, 971 (1997) (in Japanese).

[4] N. Hirata, et al., Thermal Management System of Lithium-ion Battery for The NISSAN ALTRA-EV, EVS-14 (1997).

[5] H. Horie, et al., Development of A high Power Lithium-Ion Battery System for HEV, EVS14 (1997).

[6] T. Kikuchi, et al., Evaluation Tests of Nissan Hybrid Electric Vehicle, EVS14 (1997).

[7] H. Horie, et al., Development of a PHEV Power Supply System using High-power Lithium-ion Batteries, Preprints of the Autumn Scientific Lecture Series of JSAE, 90, 38633 (1998) (in Japanese).

[8] T. Abe, et al., Development of a Cooling System for High-power Lithium-ion Batteries for HEV Application, Preprints of the Spring Scientific Lecture Series of JSAE, (1998) (in Japanese).

[9] S. Kitada, et al., Development of a Parallel HEV System Incorporating a CVT, EVS-15 (1998).

[10] Y. Tanjo, et al., Abstract for the 40th Battery Forum, 2C04 (1999) (in Japanese).

[11] H. Horie, et al., Development of a High-Power Lithium-ion Battery for Parallel HEVs, EVS16 (1999).

[12] Y. Ohsawa, et al., Abstract for the 41st Battery Forum, 3 C15 (2000) (in Japanese).

[13] M. Origuchi, et al., Lithium-ion Battery Application to the Tino Hybrid, EVS17 (2000).

[14] E. Inada, et al., Development of a High-Performance Hybrid Electric Vehicle, "Tino Hybrid", EVS17 (2000).

[15] F. Saito, et al., Verifications of the Battery Adaptation in the Market and Estimation of Battery Life for EV, HEV, EVS18 (2001).

[16] K. Watanabe, et al., Study of a High-Power Lithium-ion Battery, The206th Meeting of The Electrochemical Society, Abs. 441 (2004).

[17] H. Horie, et al., Compact Lithium-ion Battery, JSAE Review, Vol. 58, No. 7 (2004) (in Japanese).

[18] O. Shimamura, et al., Development of a Compact, High-power Lithium-ion Battery System, Transactions of JSAE, Vol. 36, No. 4, pp. 138-139 (2004) (in Japanese). 
[19] H. Horie, et al., Development of Ultra-high Power Lithium-ion Batteries, IMLB-12 (12th International Meeting of Lithium Batteries), Abs.50 (2004).

[20] O. Shimamura, et al., Development of a High Power Compact Lithium-ion Battery System, EVS-21 (2005).

[21] T. Abe, et al., Research and Development Work on Lithium-ion Battery for Environmental Vehicle, To be presented at the Autumn Scientific Lecture Series of JSAE, Sept. 2006 (in Japanese).

[22] T. Abe, et al., Simulation of a High-Power Lithium-ion Battery, The 200th Meeting of The Electrochemical Society, Abs. 133 (2001).

[23] T. Abe, et al., Abstract for the 42nd Battery Forum, 3C05 (2001) (in Japanese)

\section{BIOGRAPHIES}

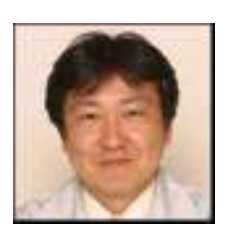

\section{Osamu Shimamura}

NISSAN MOTOR CO., LTD.

Phone:+81-46-867-5192 Fax:+81-46-867-3611

E-mail: o-shimamura@mail.nissan.co.jp

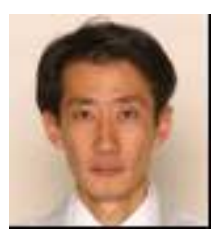

\section{Takaaki Abe}

NISSAN MOTOR CO., LTD.

Phone:+81-46-867-5192 Fax:+81-46-867-3611

E-mail:takaaki-abe@mail.nissan.co.jp

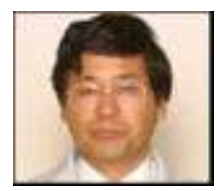

\section{Yasuhiko Ohsawa}

NISSAN MOTOR CO., LTD.

Phone:+81-46-867-5192 Fax:+81-46-867-3611

E-mail:y-oosawa@mail.nissan.co.jp

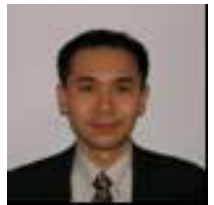

Kyoichi Watanabe

NISSAN MOTOR CO., LTD.

Phone:+81-46-867-5192 Fax:+81-46-867-3611

E-mail: kyoichi-w@mail.nissan.co.jp

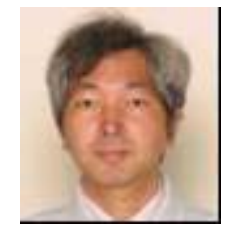

Hideaki Horie

NISSAN MOTOR CO., LTD.

Phone:+81-46-867-5192 Fax:+81-46-867-3611

E-mail: hi-horie@mail.nissan.co.jp 